\title{
Informationen über Klinische Studien werden transparent
}

\section{Zusammenfassung}

Im Portal PharmNet.Bund (www.pharmnet-bund.de) sind ab sofort Informationen über in Deutschland genehmigte klinische Arzneimittelprüfungen zu finden. "Jeder kann die Daten über klinische Studien kostenfrei einsehen", begrüßte Bundesgesundheitsminister Daniel Bahr die neue Datenbank Klinische Prüfungen. "Damit stellen wir Transparenz her für Ärzte, Patienten und andere interessierte Bürger."

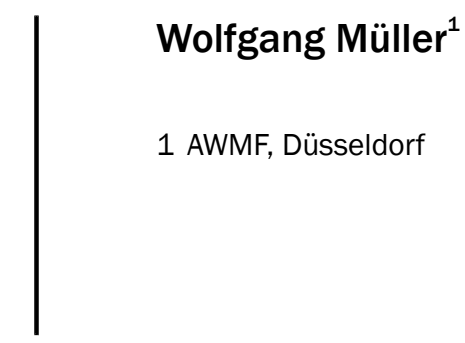

\section{Text}

Die Datenbank enthält umfangreiche Datensätze zu klinischen Prüfungen ab August 2004. Dazu gehören u.a. Informationen über:

- Sponsor (für die Prüfung verantwortliche natürliche oder juristische Person)

- Design (Aufbau, Aufteilung auf Prüfzentren, Dauer etc.)

- zu prüfende/geprüfte Arzneimittel

- Anwendungsgebiete, Ziele und Prüfungsphasen

- Personen, die in die Prüfung eingeschlossen werden sollen

Mit einer komfortablen Suchfunktion kann die Datenbank gezielt nach bestimmten Aspekten, Studienphasen, Diagnostik oder Arzneimitteln durchsucht werden.

Im Portal PharmNet.Bund sind alle in der Datenbank genutzten Begriffe ausführlich beschrieben. Die neuen Webseiten zu klinischen Prüfungen informieren zudem über die Herkunft der Daten und nach welchen Regeln diese veröffentlicht werden.

Voraussichtlich Anfang 2013 finden Sie auch die Ergebnisberichte von vielen klinischen Prüfungen in der Datenbank.

\section{Weitere Informationen im Web:}

- Klinische Prüfungen bei PharmNet.Bund:

h t t p://w w w. pharmnet-bund.de/de/ klinische-pruefungen/index.htm

- Dokumentstruktur und Datenfelder:

h t t p : / / w w w . pharm net - bund.de/de / klinische-pruefungen/veroeffentlichte-daten/index.htm

- Informationen des BfArM zu klinischen Prüfungen: http://www.bfarm.de/DE/Arzneimittel/1_vorDerZul/ klinPr/klinpr-node.html

- Informationen des PEl zu klinischen Prüfungen: http://www.pei.de/cln_236/nn_163030/DE/infos/ pu / genehmigung-klinische-pruefung/ genehmigung-klinische-pruefung-node.html?_nnn= true
- Ergebnisberichte klinischer Prüfungen bei PharmNet.Bund:

http://www.pharmnet-bund.de/de/ergebnisberichte/ index.htm

Mit PharmNet.Bund entsteht ein integriertes ArzneimittelInformationssystem, das die bundesweit vorliegenden amtlichen Daten über zugelassene Arzneimittel in Deutschland zentral zur Verfügung stellt. PharmNet.Bund wird als zentrale Plattform Patienten, Ärzten und Apothekern Gelegenheit zur zuverlässigen Recherche bieten, den Behörden effiziente Bearbeitungsmöglichkeiten und der pharmazeutischen Industrie komfortable Vorlagemöglichkeiten, z.B. in Zulassungsverfahren.

Quelle: Gemeinsame Pressemitteilung des Bundesministeriums für Gesundheit (BMG) und des Deutschen Instituts für Medizinische Dokumentation und Information (DIMDI)

\section{Korrespondenzadresse:}

M.A. Wolfgang Müller

AWMF-Geschäftsstelle, Ubierstr. 20, 40223 Düsseldorf office@awmf.org

\section{Bitte zitieren als} transparent. GMS Mitt AWMF. 2012;9:Doc24.

DOI: 10.3205/awmf000272, URN: urn:nbn:de:0183-awmf0002721

Artikel online frei zugänglich unter

http://www.egms.de/en/journals/awmf/2012-9/awmf000272.shtml

Eingereicht: 03.12.2012

Veröffentlicht: 05.12.2012

\section{Copyright}

(C2012 Müller. Dieser Artikel ist ein Open Access-Artikel und steht unter den Creative Commons Lizenzbedingungen

(http://creativecommons.org/licenses/by-nc-nd/3.0/deed.de). Er darf vervielfältigt, verbreitet und öffentlich zugänglich gemacht werden, vorausgesetzt dass Autor und Quelle genannt werden.
Müller W. Informationen über Klinische Studien werden 